\title{
Application of Receiver Operating Characteristic (ROC) Curve to Determine the Diagnostic Ability of A Validated Ten - Item Questionnaire (SS - 10) In Estimating the Prevalence of Sensitive Skin in Hong Kong Population
}

\author{
Kam Tim Michael Chan *a, Amy Ho Nam Cheung ${ }^{b}$ \\ ${ }^{a}$ Specialist in Dermatology, Hong Kong Academy of Medicine, Hong Kong SAR, China \\ ${ }^{\mathrm{b}} \mathrm{PhD}$ (University of Edinburgh), United Kingdom
}

*Corresponding author: Kam Tim Michael Chan. 2128-29, Pioneer Centre, 750, Nathan Road, Mong Kok, Kowloon, Hong Kong SAR, China; E-mail: pioneerskin@ymail.com

Received 18 June 2019;

Accepted 05 July 2019;

Published 10 July 2019

\begin{abstract}
Sensitive skin is a complex skin condition with patients presented mainly subjective neurological symptoms. Prevalence of sensitive skin across populations vary from $13 \%$ in Chinese cities to a three-fold higher in American and European countries. Our study aims to develop a cutoff value using the Receiver Operating Characteristics curve in clinical sample in Hong Kong and examine the prevalence of sensitive skin in a community sample across five districts of Hong Kong. Method: The first group of participants consisted of a total of 1,111 new clinic attendees in a local clinic in Kowloon area of Hong Kong. The second group of data was collected from 500 community samples across 5 areas of Hong Kong, with the geographic characteristics ranging from highly to less populated. Participants filled in a questionnaire which contained their demographic information as well as the 10-item version of Sensitive Skin Scale (SS-10). For the clinical sample, a dermatologist diagnosed all the participants for sensitive skin and identified 84 cases (7.56\%) of true sensitive skin. Results and conclusion: The Area Under the Curve (AUC) of 0.866 of the ROC curve suggested a good diagnostic ability of SS-10 in population of Hong Kong. A cutoff value of 25.5 with a sensitivity of $91.7 \%$ and specificity of $75.5 \%$ gave rise to $11.4 \%$ of prevalence of sensitive skin in the community sample, which is coherent with that in Mainland China. The study may have significant clinical implications for SS-10 to be a standardized and cost-effective screening tool in Asian populations.
\end{abstract}

Keywords: Sensitive skin, prevalence, 10-items version of the Sensitive Scale, cutoff value, ROC curve, Area Under the Curve

\section{Introduction}

Sensitive skin is a skin condition presented mainly with subjective neurological symptoms with minimal signs. ${ }^{[1,2]}$ It is reported to be a common global skin condition with etiology and pathogenesis still unclear. ${ }^{[2-6]}$ The absence of a definitive diagnostic test made epidemiological and surveillance study especially its prevalence estimation difficult. ${ }^{[7]}$ Diagnosis relied on the vigilance of doctors diagnosing the condition during face to face patient consultation in clinic settings. Published prevalence data mostly obtained from clinics, phone surveys or mail out questionnaires. ${ }^{[8-10]}$ Community surveys carried out based on self-reported four-point scale: very sensitive; moderately sensitive; not very sensitive and not sensitive at all may be subjectively interpreted by individuals depending on the traits of the interviewees, states and severity of the disease during interviews. ${ }^{[4,5,11-16]}$ For a subjective neurological disease like sensitive skin, self- reported semi-quantitative questionnaire survey may yield over reporting and inflating prevalence due to false positivity rate. In the documented literatures, sensitive skin was reported to have an average prevalence rate of $36.9 \%$ worldwide especially in Europe and United States while an average of $13.0 \%$ was reported in the community study in three major metropolitan cities in China. ${ }^{[17,12]}$ Another study consisting of a smaller sample predominately of female showed a prevalence of $23 \%$ in the latter. ${ }^{[18]} \mathrm{A}$ wide discrepancy was seen which has not been fully explained except a different screening method. ${ }^{[19]}$

As sensitive skin is defined by abnormal sensations in response to a variety of factors, the best method to diagnose sensitive skin is the use of scales. ${ }^{[17,19]}$ The 10-items version of the Sensitive Scale (SS -10) (Appendix 1a) assessing the severity of sensitive skin with 
good content validity which captures the core symptoms of sensitive skin was developed by Misery. ${ }^{[19]}$ The questionnaire has been tested in 11 countries globally and is useful in measuring the severity of sensitive skin. The scale possesses a high internal consistency (Cronbach's alpha $=0.94)$. It also has a significant and good correlation with Dermatology Life Quality Index (DLQI) (r $=.57, \mathrm{p}<0.0001)$ and has been useful in different geographical settings. However, its cutoff level or value in diagnosing sensitive skin has never been reported. Without establishing a cutoff value, the scale could not be standardized and its predictability and diagnostic power dependent on sensitivity and specificity will be compromised. This limit its long - term use as an effective and accurate self -reporting epidemiological diagnostic and surveillance tool of sensitive skin across different populations of the world. In fact, the paper suggested individuals scored from 20 to 60 in SS -10 could be identified as sensitive skin despite no scientific explanation given; albeit; further studies are suggested by the authors. ${ }^{[19]}$

In view of this, we attempt to develop a cutoff value in a clinical sample of Hong Kong by plotting a Receiver Operating Characteristic (ROC) curve based on sensitive skin data collected locally to select a cut off value with high sensitivity and predictability and applied it in an epidemiological community study to estimate the prevalence of sensitive skin in the local population of Hong Kong.

\section{Method}

The first group of data came from a clinic based prospective study carried out during the period of 1st May 2018 to 30 April 2019 in a private clinic located in the Centre of Kowloon district, Hong Kong. All newly attended patients during this study period were recorded and consulted by a dermatologist. The proportion of clinically diagnosed sensitive skin patients in the private clinic setting, demographic characteristics including age, gender, occupation, marital status, site of involvement, medical history and concomitant skin dermatosis were recorded by a dermatologist. The clinical diagnosis of sensitive skin is based on the definition suggested by International Forum for the Study of Itch (IFSI): sensitive skin is a syndrome defined by the occurrence of unpleasant sensations (stinging, burning, pain, pruritus, and tingling sensations) in response to stimuli that normally should not provoke such sensations. These unpleasant sensations cannot be explained by lesions attributable to any skin disease. ${ }^{[21]}$ Prior to the consultation, the patients were asked voluntarily to complete the SS-10. Consent was obtained by completing and returning the questionnaire A translated Chinese version of SS - 10 was used. (Appendix 1b) SS -10 score was recorded and compared with the clinically diagnosis of sensitive skin. The score was also recorded in the subsequent follow up consultation to monitor progress of the disease after management with counselling and emollients only. A total of 1,111 cases were recruited and 84 cases were diagnosed as true sensitive skin and 1,027 were diagnosed as true negative sensitive skin cases. $(\text { Table } 1)^{[20]}$

Table 1

\begin{tabular}{|l|l|}
\hline $\begin{array}{l}\text { Clinical diagnosis of sensitive skin in clinic } \\
\text { setting made by dermatologist based on the } \\
\text { IFSI definition }\end{array}$ & $\begin{array}{l}\text { Number of } \\
\text { Patients }\end{array}$ \\
\hline True Positive Cases & 84 \\
\hline True Negative Cases & 1027 \\
\hline Total & 1111 \\
\hline
\end{tabular}

The second group of data consisted of epidemiological data from a community study on the prevalence of sensitive skin in Hong Kong using the validated Sensitive Scale -10 (SS-10) Questionnaire.

In January 2018, a total of 500 subjects were recruited using quota sampling method in different geographical areas from more densely populated commercial areas to more spacious sub-urban areas. Having covered a comprehensive geographic feature of Hong Kong at various locations in the sampling increased the representativeness of the studied sample to be generalized to the overall Hong Kong population. Two of the locations were in Kowloon peninsula (Mong Kok, Tsim Sha Tsui), one in Hong Kong island (Causeway Bay), two in the rest of Hong Kong which are less densely populated than the former three (Tsuen Wan and Shatin). Participants were interviewed face-to-face by volunteers, who have no prior knowledge of sensitive skin, on the streets in these geographical locations in Hong Kong. The participation was voluntary. Consent was given to the interviewer when participants agreed to complete the questionnaire and the anonymity of participants was enforced. The questionnaire administrated included demographic information (gender, age, ethnicity, marital status, number, gender and age of children, and concomitant skin diseases), following which was the Chinese version of SS-10.

Using the SPSS 22 software, the Receiver Operating Characteristic (ROC) curve analysis was performed to estimate the area under the curve (AUC) and the cut off value with high sensitivity and specificity.

\section{Results}

As show in Table 2, a cutoff value of $>25.5$ yielded a sensitivity of $91.7 \%$ and specificity of $75.5 \%$ in the clinical data $(\mathrm{N}=1111)$. An area under the curve of 0.866 suggested that SS-10 had good diagnostic ability (Figure 1). From the second group of data, a prevalence of $11.4 \%$ was documented in the community population with a cut -off value of 25.5 give rise to a prevalence of $11.4 \%$. $(\mathrm{N}=500)$.

Table 2

\begin{tabular}{|l|l|l|}
\hline \multicolumn{3}{|c|}{ Coordinates of curve } \\
\multicolumn{3}{|c|}{ Test Result Variable(s): Ss-total score } \\
\hline $\begin{array}{l}\text { Positive if Greater } \\
\text { Than or Equal To }\end{array}$ & Sensitivity & 1-Specificity \\
\hline-1.00 & 1.000 & 1.000 \\
.50 & 1.000 & .793 \\
1.50 & 1.000 & .761 \\
2.50 & 1.000 & .732 \\
3.50 & 1.000 & .701 \\
4.50 & 1.000 & .670 \\
5.50 & 1.000 & .627 \\
6.50 & 1.000 & .602 \\
7.50 & 1.000 & .572 \\
8.50 & 1.000 & .535 \\
9.50 & 1.000 & .510 \\
10.50 & 1.000 & .477 \\
11.50 & 1.000 & .463 \\
12.50 & 1.000 & .440 \\
13.50 & 1.000 & .426 \\
14.50 & 1.000 & .406 \\
15.50 & 1.000 & .381 \\
16.50 & 1.000 & .367 \\
17.50 & 1.000 & .351 \\
18.50 & 1.000 & .337 \\
\hline
\end{tabular}




\begin{tabular}{|l|l|l|}
\hline 19.50 & 1.000 & .322 \\
20.50 & .988 & .306 \\
21.50 & .964 & .285 \\
22.50 & .952 & .277 \\
23.50 & .940 & .262 \\
24.50 & .917 & .253 \\
25.50 & .917 & .245 \\
26.50 & .881 & .234 \\
27.50 & .845 & .224 \\
\hline
\end{tabular}

Figure 1

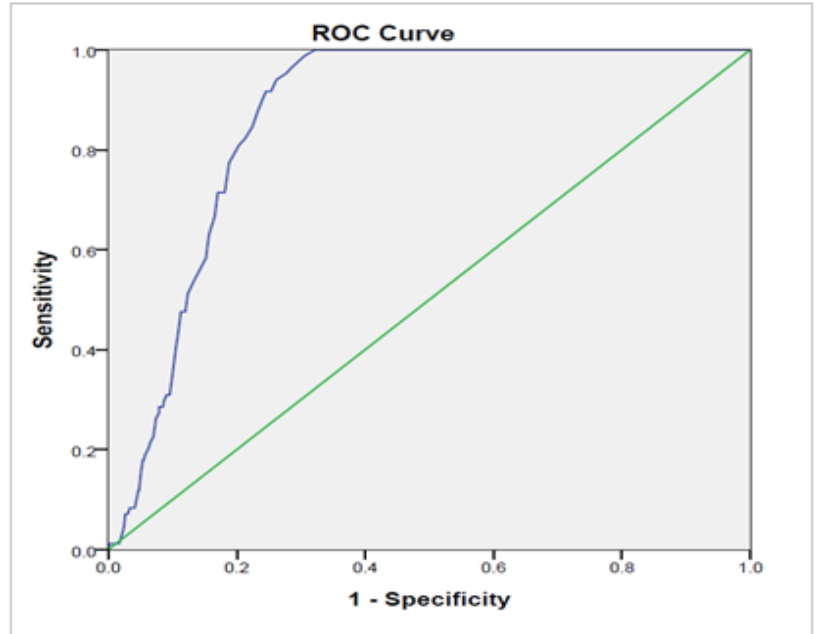

\begin{tabular}{|l|l|l|l|}
\hline $\begin{array}{l}\text { Area Under } \\
\text { the Curve }\end{array}$ & $\begin{array}{l}\text { Standard } \\
\text { Error }\end{array}$ & $\begin{array}{l}\text { Asymptotic 95\% Confidence } \\
\text { Interval }\end{array}$ \\
\cline { 3 - 4 } & & Lower Bound & Upper Bound \\
\hline 0.866 & 0.012 & 0.843 & 0.889 \\
\hline
\end{tabular}

Table 3

\begin{tabular}{|l|l|l|}
\hline $\mathbf{N}=$ number & Valid & $\mathbf{5 0 0}$ \\
\hline & Missing & 0 \\
\hline
\end{tabular}

\begin{tabular}{|l|c|c|c|c|}
\hline & Frequency & Percent & $\begin{array}{c}\text { Valid } \\
\text { Percent }\end{array}$ & $\begin{array}{c}\text { Cumulative } \\
\text { Percent }\end{array}$ \\
\hline Valid .00 & 443 & 88.6 & 88.6 & 88.6 \\
\hline $\mathbf{1 . 0 0}$ & 57 & 11.4 & 11.4 & 100.0 \\
\hline Total & 500 & 100.0 & 100.0 & \\
\hline
\end{tabular}

\section{Discussion}

Since the development of the SS-10, the total score has been used as a continuum to indicate the severity of skin sensitivity while diagnostic and screening properties are absent due to a lack in cutoff value. In this paper, the cutoff value of SS-10 is developed through plotting a ROC curve in clinical population in Hong Kong, giving rise to a prevalence of $11.4 \%$ that is coherent with the proportion of diagnosis by a dermatologist in the clinical study by Chan. ${ }^{[20]}$

A Receiver Operating Characteristic (ROC) Curve is an established statistical method to compare diagnostic tests. ROC Curve had been shown efficacious and applicable in many areas of science including medicine. It was originally developed during the Second World War by the Allies as part of the radar system to analyse data to differentiate between enemy aircrafts and signal noise produced by flocks of birds. As the sensitivity of the receiver increased, so did the number of false positives. We experiment to compare the dermatologist clinical diagnosis of sensitive skin based on the IFSI definition in a clinic base setting to the validated SS - 10 Questionnaire scores in the community setting in Hong Kong.

The ROC is a plot of the true positive rate against the false positive rate. The ROC curve enabled the researcher ability in the following areas:

1. The relationship between sensitivity and specificity. As illustrated in the ROC curve, an increase in sensitivity will result in a decrease in specificity; the converse is equally true; a decrease in sensitivity results in an increase in specificity.

2. To verify accuracy; the closer the graph to the top and left-hand borders, the more accurate the test is. Similarly, the test is less accurate when the graph is closer to the diagonal. An ideal perfect test; which is unlikely to exist in real world; would go straight from zero up the top-left corner and then straight across the horizontal.

3. To aid researcher manually to select the best cutoff value to yield the maximum sensitivity and specificity which is usually at least 0.8 or greater.

The area under the curve of the ROC curve is important to exemplify statistically whether the test used to compare is a good test of diagnostic value. The greater the area under the curve, the more accurate the test. A perfect test has an area under the ROC curve of 1 and a less useful test will be under 0.5 . In our case, the area under the curve of our derived ROC curve $=0.866(95 \%$ Confidence Interval: $0.843-0.889)$ indicate the SS - 10 Questionnaire has a good diagnostic ability in our community setting study of sensitive skin in Hong Kong. (Table 4)

Table 4: The relationship between the area under the curve (AuC) and diagnostic ability of the test

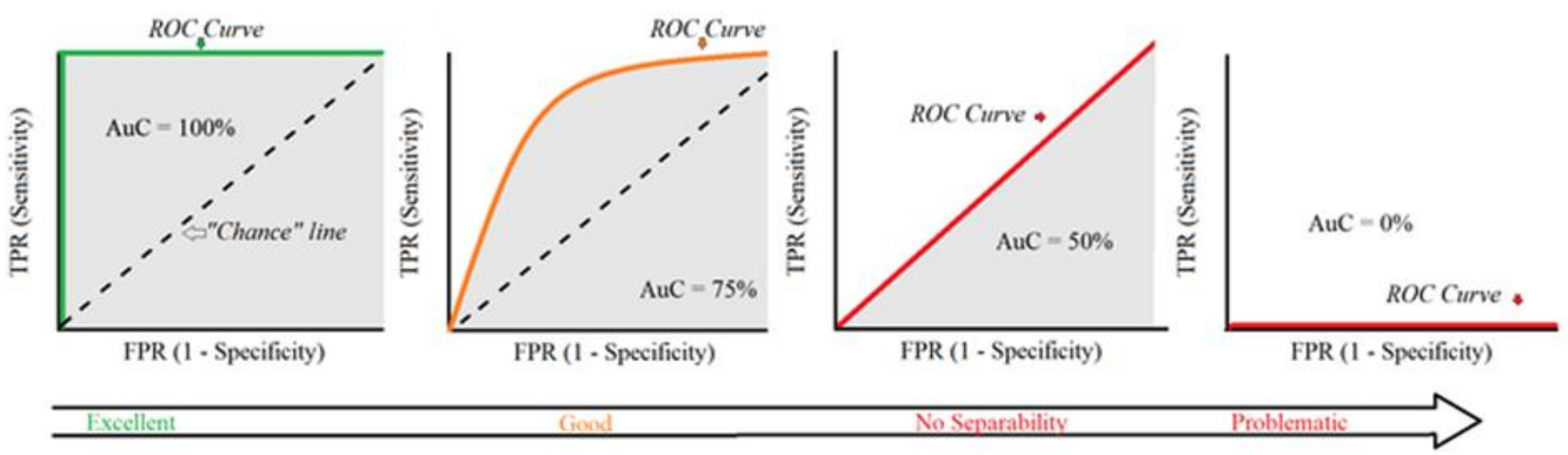


(The green line is the perfect test, the orange line indicated an above 0.5 or $50 \%$ chance of an accurate test; i.e.; good test, the red line is the diagonal below which is less than 0.5 or $50 \%$ chance of having an accurate test; i.e.; a bad test in diagnosis. $\mathrm{TPR}=$ true positive rate; FPR $=$ false positive rate $=1-$ Specificity)

In the above study; using a validated 10 item sensitive scale comparing with dermatologist diagnosed true positive case of sensitive skin; we were able to estimate a more precise and accurate prevalence of sensitive skin in the community of Hong Kong. The results obtained may be more valid and reliable over time than the previously used four items questionnaire based on asking the subjects to rate their skin as "very sensitive", "sensitive", "slightly sensitive" or "not sensitive" which may be subjected to various interpretations in different geographical areas, cultures, languages and context. The ROC curve with a cutoff of at least 0.8 or higher may reduce false positivity rate and avoid overreporting. Moreover, by adjusting the likelihood ratio or cutoff value; the result may be more flexibly reflecting the prevalence of the condition in the population and to determine whether a test result usefully changes the probability that a condition (such as a disease state) exists.

Our estimated prevalence of $11.4 \%$ is much lower than that reported in the literature with an average of $36.9 \%$ but comparable to the prevalence of $13 \%$ of sensitive skin estimated by the probability cluster sampling methods in the metropolitan cities of China. Our paper is not aiming at investigating the underlying reasons for the difference observed but the high trade off of a high sensitivity rate and specificity rate may well select the "very sensitive" and " rather very sensitive" individuals in the populations who indeed are the one diseased with sensitive skin based on the clinical definition of IFSI. The high prevalence reported in the literature may only select those who have mild or occasional symptoms of sensitive skin under different circumstances but not specific enough to be diagnosed as a disease condition. It would be of interest to apply the ROC curve to determine the diagnostic ability of the validated Ten - Item Questionnaire (SS - 10) in populations in other parts of the world and compare the previously reported sensitive skin prevalence based on the four items questionnaire which is worth further investigation.

There are limitations of ROC curve like the use of dissimilar datasets in one ROC plot is a commonly reported error when ROC is used for comparing multiple classifiers. One ROC curve with several ROC points are drawn in one plot. The comparison between them is valid only when the classifiers are evaluated on either a single dataset or multiple datasets that are almost identical among each other in terms of their data size and positive: negative ratio. The other limitation is that ROC becomes less powerful when used with imbalanced datasets. One effective approach to avoid the potential issues with imbalanced datasets is using the early retrieval area, which is a region with high specificity values in the ROC space. Checking this area is useful to analyse the performance with fewer false positives (or small false positive rate). (Figure 1)

\section{Conclusion}

The current study established the cutoff value of SS-10 on a clinical data in Hong Kong. It possessed good diagnostic power and yielded $91.7 \%$ of sensitivity and $75.5 \%$ of specificity with AUC of 0.866 . Using the cutoff value of 25.5 the study reported a prevalence of $11.4 \%$ in community sample in Hong Kong, suggesting its ability to distinguish individuals with and without skin sensitivity. The study has significant clinical implications that points to wider application of SS-10 in Asian populations as a screening tool for sensitive skin.

\section{References}

[1] Misery L, Loser K, Stander S. Sensitive skin. JEADV 2016; 30(Suppl.1), 2-8.

[2] Stander S. Sensitive skin - a global challenge with upcoming solutions. Editorial. JEADV 2016; 30 (Suppl1), 1.

[3] Misery L, Myon E, Martin N, Verriere F, Nocera T, Taieb C. Sensitive skin in France: an epidemiological approach. Ann Dermatol Venereal. 2005; 132:425-9.

[4] Misery L, Boussetta S, Nocera T, Perez-Cullell N, Taieb C. Sensitive skin in Europe. J Eur Acad Dermatol Venereol. 2009; 23: 376-81.

[5] Misery L, Sibaud V, Merial-Kieny C, Taieb C. Sensitive skin in the American population: Prevalence, clinical data, and role of the dermatologist. Int J Dermatol 2011; 50(8):961 - 967.

[6] Marriott M, Holmes J, Peters L, Coper K, Rowson M, Basketter DA. The complex problem of sensitive skin. Contact dermatitis. 2005;53;93-99.

[7] Chan KTM. Clinical Review on Sensitive Skin: History, Epidemiology, Pathogenesis and Management. J Clin Exp Dermatol Res. 2018; 9: 453.

[8] Saint-Martory C, Roguedas-Contios AM, Sibaud V, Degouy A, Schmitt AM, Misery L. Sensitive skin is not limited to the face. Br J Dermatol. 2008; 158(1):130-133.

[9] Farage M. Sensitive skin in China. Journal of Cosmetics and Dermatological Sciences and applications. 2012; 2(3); 184-95.

[10] Jourdain R, de Lacharriere O, Bastien P, Maibach HI. Ethnic variations in self-perceived sensitive skin: Epidemiological survey. Contact Dermatitis. 2002;46(3)162-9.

[11] Kamide R, Misery L, Perez-Cullel n, Sibaud V, Taieb C. Sensitive skin evaluation in the Japanese population. J Dermatol 2013; 40:177-81.

[12] Xu F, Yan S, Wu M, Li F, Sun Q, Lai W, Shen X, Rahhali N, Taieb C, Xu J. Self-declared sensitive skin in China: A community -based study in three top metropolises. J Eur Acad Dermatol Venereol. 2013; 27: 370-5.

[13] Farage M. Sensitive skin in China. Journal of Cosmetics and Dermatological Sciences and applications. 2012; 2(3); 184-95.

[14] Jourdain R, de Lacharriere O, Bastien P, Maibach HI. Ethnic variations in self-perceived sensitive skin: Epidemiological survey. Contact Dermatitis. 2002;46(3)162-9.

[15] Loffler H, Dickel H, Kuss O, Diepgen TL, Effendy I. Characteristics of self-estimated enhanced skin susceptibility. Acta Dermato-venereologica. 2001;81(5): 343-6.

[16] Farage MA. How do perceptions of sensitive skin differ at different anatomical sites? An epidemiological study. Clinical and Experimental Dermatology.2009;3498); e521-30. 
[17] Honari G, Andersen RM, Maibach HI. Sensitive skin syndrome. Second Edition. Chapter 2, p12.

[18] Farage M. Sensitive skin in China. Journal of Cosmetics and Dermatological Sciences and applications. 2012; 2(3); 184-95.

[19] Misery L, Jean-Decoste C, Mery S, Georgescu V, Sibaud V. A New Ten -item Questionnaire for assessing Sensitive Skin. The Sensitive Scale 10. Acta Derm Venereol. 2014, 94: 635 -639.

[20] Chan KTM. A Prospective Study on the Characteristics of Sensitive Skin Syndrome in Chinese Patients
Attending a Private Clinic-Based Setting in Hong Kong. J Clin Exp Dermatol Res. 2019, 10:498. doi: 10.24105/2155-9554.10.498.

[21] Misery L, Stander S, Szepietowski JC, Reich A, Wallengren J, Evers AWM, Takamori K, Brenaut E, Le Gall-lanotto C, Fluhr J, Berardesca E, Weisshaar E. Definition of sensitive skin: An expert position paper from the special interest group on sensitive skin of the international forum for the study of itch. Acta Derma Venereol; 2017; 97(1), 4-6. 


\section{$\underline{\text { Appendix 1a and 1b }}$}

Supplementary material to article my L. Miserey et al. "A New Ten-Item Questionnaire For Assessing Sensitive Skin:The Sensitive Scale-10" DEGREE OF OVERALL SKIN IRRITATION DURING THE PAST 3 DAYS

Using a vertical line, indicate the symptoms felt during the past 3 days on the horizontal line $(0=$ absence of irritaion, $10=$ intolerance irritation)

A Importent To be completed by the patient

Skin irritation

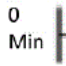

SEVERITY OF SKIN CONDITION DURING THE PAST 3 DAYS

Please indicate the intensity of each of the following symptoms during the past 3 days $0=$ zero intensity, $10=$ intolerable intensity): darken one number between 0 and 10

$$
\text { A Importent To be completed by the patient }
$$

Skin Condition felt

Tingling

Burning

की कर

Sensations of heart

Thoughts

Itching

Pain

General discomfort

Hot flashes

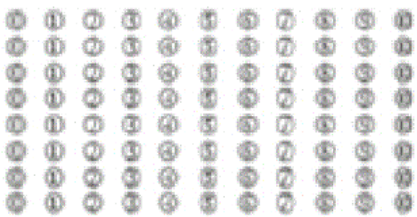

Visible Skin Condition

Redness

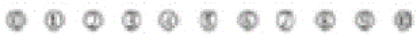

Fig. S2 English version of Sensitive Scale-10
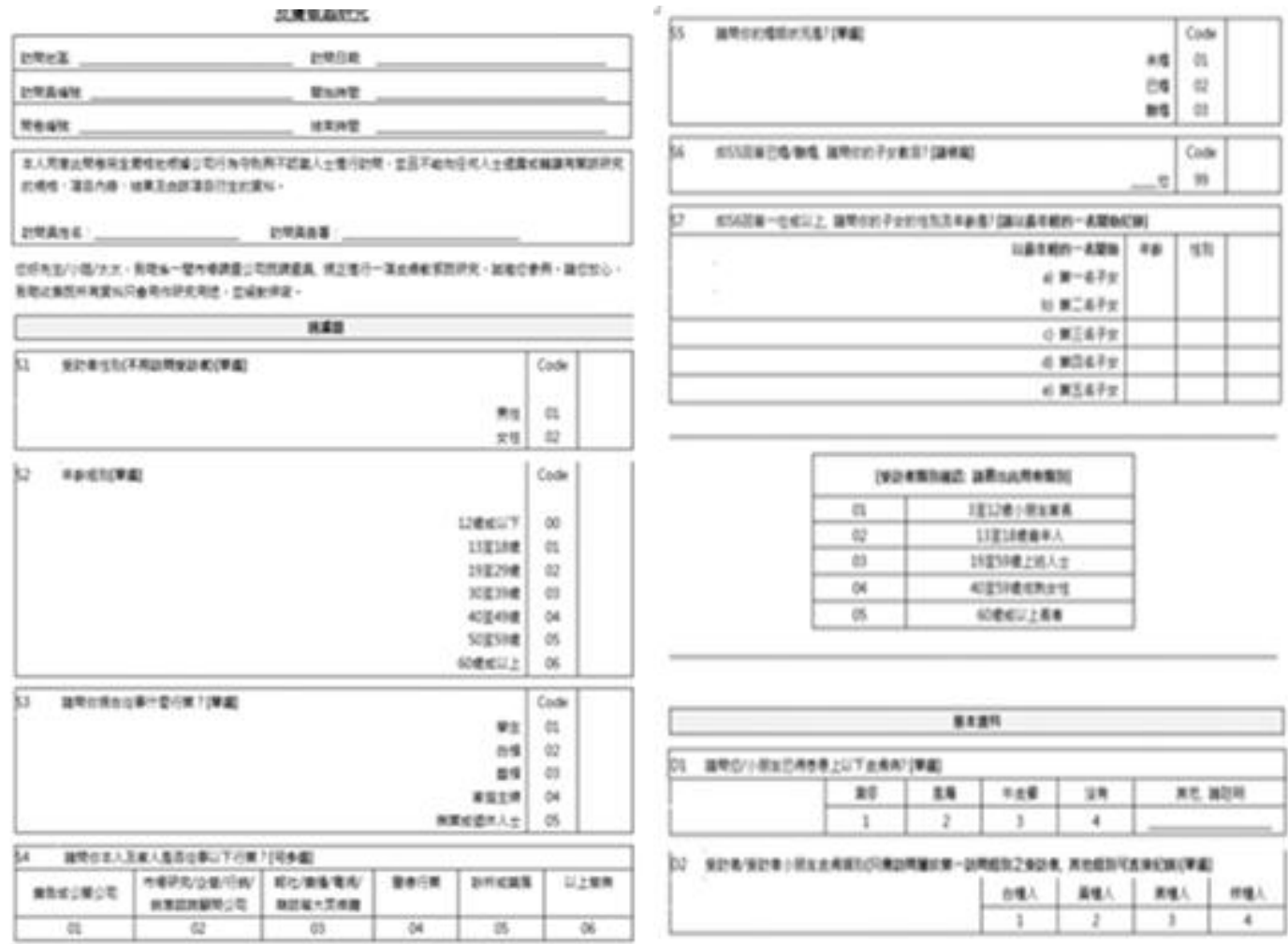

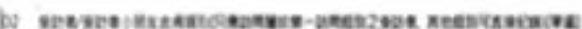

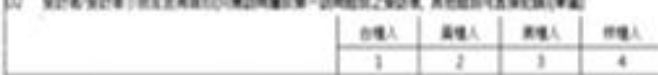




\section{皮痛䜿原量表-10}

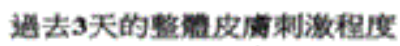

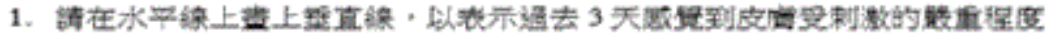

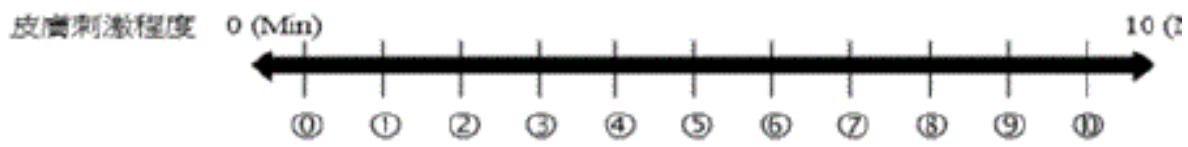

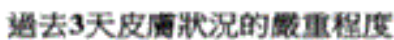

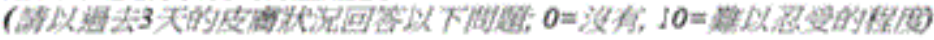

\begin{tabular}{|c|c|c|c|c|c|c|c|c|c|c|}
\hline 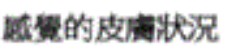 & & & & & & & & & & \\
\hline 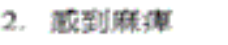 & (2) & (1) & (2) & (3) & (4) & (5) & (6) & (7) & (8) & (9) \\
\hline 3. 武到火塻 & (1) & (1) & (2) & (2) & (4) & (5) & (2) & (8) & (3) & (2) \\
\hline 4. 牫到灼知 & (1) & (1) & (2) & (3) & (4) & (5) & (4) & (2) & (8) & (9) \\
\hline 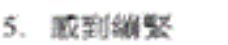 & (1) & (1) & (2) & (3) & (4) & (5) & (4) & (8) & (3) & (9) \\
\hline 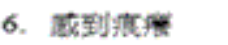 & (1) & (1) & (2) & (2) & (4) & (5) & (6) & (2) & (8) & (9) \\
\hline 7. 成到湔起 & (1) & (1) & (2) & (2) & (4) & (5) & () & (1) & (3) & (2) \\
\hline 8. 成到不辞脂 & (Q) & (1) & (2) & (3) & (4) & (5) & (5) & (7) & (3) & (9) \\
\hline 9. 国到: & (1) & (1) & (2) & (3) & (4) & (5) & (6) & (7) & (8) & (9) \\
\hline
\end{tabular}

需見的皮厧状况

10. 泛紅

(1) (1) (2)

(2) (4) (4)

(2)

(3) (2) (1)

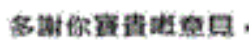

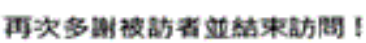

\section{Appendix 2: Combination of sensitivity and 1-specificity according to various cut off values}

\begin{tabular}{|l|l|l|}
\hline Positive if Greater Than or Equal To $^{\mathrm{a}}$ & Sensitivity & 1 - Specificity \\
\hline-1.00 & 1.000 & 1.000 \\
\hline .50 & 1.000 & .793 \\
\hline 1.50 & 1.000 & .761 \\
\hline 2.50 & 1.000 & .732 \\
\hline 3.50 & 1.000 & .701 \\
\hline 4.50 & 1.000 & .670 \\
\hline 5.50 & 1.000 & .627 \\
\hline 6.50 & 1.000 & .602 \\
\hline 7.50 & 1.000 & .572 \\
\hline 8.50 & 1.000 & .535 \\
\hline 9.50 & 1.000 & .510 \\
\hline 10.50 & 1.000 & .477 \\
\hline 11.50 & 1.000 & .463 \\
\hline 12.50 & 1.000 & .440 \\
\hline 13.50 & 1.000 & .426 \\
\hline 14.50 & 1.000 & .406 \\
\hline 15.50 & 1.000 & .381 \\
\hline 16.50 & 1.000 & .367 \\
\hline 17.50 & 1.000 & .351 \\
\hline 18.50 & 1.000 & .337 \\
\hline 19.50 & 1.000 & .322 \\
\hline 20.50 & .988 & .306 \\
\hline 21.50 & .964 & .285 \\
\hline & & \\
\hline
\end{tabular}


International Journal of Innovative Research in Medical Science (IJIRMS)

\begin{tabular}{|c|c|c|}
\hline 22.50 & .952 & .277 \\
\hline 23.50 & .940 & .262 \\
\hline 24.50 & 9.917 & .253 \\
\hline 25.50 & .917 & .245 \\
\hline 26.50 & .881 & .234 \\
\hline 27.50 & .845 & .224 \\
\hline 28.50 & .821 & .212 \\
\hline 29.50 & .810 & .204 \\
\hline 30.50 & .774 & .188 \\
\hline 31.50 & .714 & .181 \\
\hline 32.50 & .714 & .170 \\
\hline 33.50 & .667 & .166 \\
\hline 34.50 & .631 & .157 \\
\hline 35.50 & .583 & .152 \\
\hline 36.50 & .560 & .142 \\
\hline 37.50 & .536 & .132 \\
\hline 38.50 & .512 & .124 \\
\hline 39.50 & .476 & .120 \\
\hline 40.50 & .476 & .113 \\
\hline 41.50 & .429 & .108 \\
\hline 42.50 & .405 & .105 \\
\hline 43.50 & .345 & .099 \\
\hline 44.50 & .310 & .095 \\
\hline 45.50 & .310 & .091 \\
\hline 46.50 & .298 & .087 \\
\hline 47.50 & .286 & .085 \\
\hline 48.50 & .286 & .079 \\
\hline 49.50 & .274 & .079 \\
\hline 50.50 & .262 & .074 \\
\hline 51.50 & .226 & .070 \\
\hline 52.50 & .214 & .065 \\
\hline 53.50 & .202 & .062 \\
\hline 54.50 & .190 & .057 \\
\hline 55.50 & .179 & .055 \\
\hline 56.50 & .179 & .054 \\
\hline 57.50 & .155 & .051 \\
\hline 58.50 & .131 & .049 \\
\hline 59.50 & .119 & .048 \\
\hline 60.50 & .119 & .047 \\
\hline 61.50 & .083 & .041 \\
\hline 62.50 & .083 & .039 \\
\hline 63.50 & .083 & .037 \\
\hline 64.50 & .083 & .035 \\
\hline 65.50 & .083 & .034 \\
\hline 66.50 & .083 & .033 \\
\hline 67.50 & .071 & .030 \\
\hline 69.00 & .071 & .027 \\
\hline 70.50 & .071 & .026 \\
\hline 71.50 & .048 & .024 \\
\hline 72.50 & .036 & .022 \\
\hline 74.00 & .024 & .019 \\
\hline 76.00 & .012 & .017 \\
\hline 77.50 & .012 & .016 \\
\hline 78.50 & .012 & .014 \\
\hline 79.50 & .012 & .012 \\
\hline 80.50 & .012 & .011 \\
\hline 81.50 & .012 & .010 \\
\hline 82.50 & .012 & .009 \\
\hline 83.50 & .012 & .008 \\
\hline 84.50 & .012 & .006 \\
\hline 86.50 & .012 & .005 \\
\hline
\end{tabular}


International Journal of Innovative Research in Medical Science (IJIRMS)

\begin{tabular}{|l|l|l|}
\hline 89.50 & .012 & .003 \\
\hline 93.00 & .012 & .002 \\
\hline 97.00 & .012 & .001 \\
\hline 99.50 & .000 & .001 \\
\hline 101.00 & .000 & .000 \\
\hline
\end{tabular}

